\title{
Objective Measurement of Walking Activity Using Wearable Technologies in People with Parkinson Disease: A Systematic Review Protocol
}

\author{
Thomas Carlin ${ }^{a, b} \quad$ Clint Hansen ${ }^{c}$ Nicolas Vuillerme ${ }^{a, b, d}$ \\ aLaboratory AGEIS, Universitaire Grenoble Alpes, Grenoble, France; babCom Telecom4Health, Universitaire \\ Grenoble Alpes, Grenoble, France; 'Department of Neurology, Universitätsklinikum Schleswig-Holstein, Kiel,

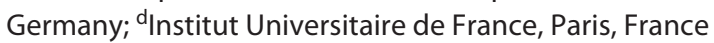

\section{Keywords}

Parkinson disease - Walking activity - Wearable devices ·

Monitoring strategy $\cdot$ Systematic review

\begin{abstract}
Introduction: Parkinson's disease (PD) is a complex neurodegenerative disease with motor and nonmotor symptoms with a multitude of disease variations and severity. Physical activity can improve the management of disease symptoms and increase patients' quality of life. Technological development of small wearable devices allows objective activity measurement such as daily step count. Objective: To synthesize ongoing and past research on objective walking activity measurements using wearable devices in patients with PD. Methods: PubMed, Cochrane, Web of Science, and PEDro database are systematically searched with no limitation on publication date. Keywords are relative to (1) the population, (2) the measurement tool, and (3) the measured outcomes. Only full-text English articles published in a peer-reviewed journal will be included. Participants do not have to undergo any type of intervention. Included studies must report an objective measurement of walking activity using wearable devices in PD patients. After an independent screening process done by 2 reviewers, data will be extracted from the articles according to the following 5 set of data: (1) the study metrics,
\end{abstract}

(2) the population characteristics, (3) the measurement tools, (4) the experimental procedure, and (5) the reported outcomes. Results: The results will contain inter alia summaries of the wearables' specifications, wearing location, and recommendations for feasible methodologies to capture daily walking activity. Discussion: This review aims to synthesize the evidence of objective walking activity assessment with wearable devices in patients with PD. It will also provide recommendations with regard to device selection and suggest key points when monitoring walking activity in this specific population.

(c) 2021 The Author(s) Published by S. Karger AG, Basel

\section{Introduction}

Parkinson's disease (PD) is a neurodegenerative disease affecting motor $[1,2]$ and nonmotor skills of patients [3]. Typical motor symptoms of the disease include bradykinesia, muscular rigidity, resting tremor, and postural instability $[1,4]$. Nonmotor or nondopaminergic symptoms emerging inevitably with the disease seem to be promising prodromal PD indicators $[3,5,6]$. These symptoms lead to an increasing level of dependence with the emergence of olfactory disturbances, autonomic dys-

karger@karger.com www.karger.com/bmh

Karger $\stackrel{\text { ' }}{5}$

GOPEN ACCESS
(C) 2021 The Author(s)

Published by S. Karger AG, Basel

This is an Open Access article licensed under the Creative Commons Attribution-NonCommercial-4.0 International License (CC BY-NC) (http://www.karger.com/Services/OpenAccessLicense), applicable to the online version of the article only. Usage and distribution for commercial purposes requires written permission.
Correspondence to:

Clint Hansen, c.hansen@ neurologie.uni-kiel.de 
function, pain, sleep fragmentation, depression, and dementia $[3,7]$. Due to the complex pathophysiology of PD $[1,3,7]$ and the wide variability of progression symptoms $[5,6,8-11]$, the diagnosis and treatment remain difficult [12]. People suffering from PD often present impaired functional abilities [13-15], a reduced level of strength $[15,16]$, and a lower level of physical activity $[17,18]$, overall leading to an increased fall risk [19-21].

Evidence suggests that physical activity may limit the progression of neurodegenerative impairment, associated with PD [22]. Aerobic training seems to slow brain degeneration with positive effects on depression [23], apathy, fatigue, daytime sleepiness, sleep, and cognition [24].

Research suggests that wearable technologies are valid methods to monitor the relationship between clinicalrating scales (e.g., MDS-UPDRS III) and walking activity in PD [25]. However, only limited information is available regarding the capabilities of wearables to monitor physical activity in PD even though this is crucial for disease management $[5,18,23,24,26-31]$. Mobility and the amount of daily walking during domestic activities [32] or as part of their daily living outside the house [33] are strongly correlated. Deficits in mobility affect not only activities of daily living but also risk of falling [34], independence [35], and motor fluctuation over time [36].

Understanding the array of solutions for instrumented monitoring of walking activity in PD will allow clinicians and researchers to better plan and implement interventions and trials. Consequently, this review aims to identify and map all available studies on the use of wearable devices for objective measurement of walking activity in people with PD.

\section{Methods}

The Preferred Reporting Items for Systematic Review and Meta-Analysis will be used to identify relevant studies [37]. We will explore the use of wearables to detect walking activity with the aim to map the state of evidence in a structured yet reflexive manner and to identify research gaps or assess the feasibility of future reviews [38]. The protocol of this current review has been registered in PROSPERO (CRD42020210866).

\section{Eligibility Criteria}

Only full-text peer-reviewed scientific original articles published in English will be included in this review. Case reports, conference abstracts, abstracts, poster, reviews, or meta-analysis will be not included. We will use the following eligibility criteria for study selection.

\section{Population}

All articles including patients with a diagnosis of PD will be included. The patients must be able to walk with or without an assistive technical aid during daily life activity. Consequently, wheelchair patients and those who are not able to walk will be not considered in this review. We will also consider data relative to control groups. Control group data contain healthy subjects or patients with other neurodegenerative diseases. Studies including animal models or mixed syndrome will be excluded.

\section{Intervention}

Participants are not required to undergo any type of intervention.

\section{Measurement Tool}

Studies will have to use wearable devices. Wearable devices may use any combination of electronic or spring-levered uni- or multiaxial accelerometer, gyroscope, magnetometer, or barometer. Other measurement tools such as heart rate monitor and thermometer will be not considered. Wearable devices have to be attached to the body, for example, belt clip or any other assistive accessory. Measurement tools can also be placed in a pocket or in a bag.

\section{Experimental Procedure}

Studies can take place in a laboratory or in a free-living environment. This review aims to synthetize the evidence on the feasibility of using wearable devices to capture walking activity pattern in patients with PD. Therefore, laboratory-based and free-living studies will be included to explain how and in what context wearable devices can be relevant. Laboratory-based studies focus on standardized assessments or the specificity of the walking pattern of PD patients while free-living-based studies will inform on the feasibility of using wearable devices to track daily walking activity. Studies assessing walking activity in a restrictive condition (predetermined course, planned outdoor tour, and sidewalk test) will also be included.

\section{Measured Outcomes}

Studies must report at least 1 output that quantifies daily walking activity (daily step count, distance traveled, and GPS track). Activity counts and physical activity level, as well as intensity of activity and energy expenditure based on counts threshold, will be not covered by this review. Metrics relative to nonmotor activities or sedentary behavior will also be excluded.

\section{Data Sources and Search Strategy}

The following 4 electronic databases will be systematically searched with no limit in the publication date: PubMed, Cochrane, Web of Science, and PEDro. A first preliminary structured search was conducted in September 2020 and will be repeated before the completion of the final review process.

Keywords related to (1) the population, (2) the measurement tool, and (3) the measured outcomes will be used. The search strategy included a combination of keywords, using the Boolean operator "AND" and "OR" and, if it is applicable, the Medical Subject Headings terms. The first category of keywords focuses the search on patients with PD and includes terms such as "idiopathic Parkinson's Disease," "Lewy Body Parkinson Disease," "Primary Parkinsonism," "Idiopathic Parkinson Disease," or "Parkinson Disease." 
The second category specifies the measurement tools that should be used to track steps in PD patients. It comprises all terms relative to wearable devices: "wearable technology," "wearable sensor," "wearable device," "ambulatory monitoring," "fitness tracker," "activity tracker," "activity monitor," "step counter," "actigraphy," "pedometer," "accelerometer," “inclinometer," "gyroscope," "inertial sensor," "inertial measurement unit," "pendant sensor," “ stopwatch," "smartphone," and "mobile phone."

The third category was designed to limit the search results to studies reporting metrics related to daily walking activity including terms such as "step count," "daily step," "steps per day," "walking activity," "walking bout," and "walking time." These 3 categories will be combined as follows for the final search: (1) AND (2) AND (3). The search fields will be restricted to the abstract, title, and keywords.

\section{Study Selection}

The title, abstract, and keyword of each article returned by our search strategy will be screened by 2 independent reviewers (T.C. and C.H.). Inclusion is based on the abovementioned selection criteria. A full-length text screening will then be performed to confirm if the potentially included studies meet the selection criteria. Finally, based on the inclusion and exclusion criteria, the eligibility of each article will be reported independently by each reviewer (T.C. and C.H.). Discrepancy will be resolved by consensus meeting using a third reviewer (N.V.) if disagreement persists.

\section{Data Extraction}

After completion of the screening process, 2 reviewers (T.C. and C.H.) will independently extract the data from each included article. Data extraction will be done following a prebuilt table including information about study metrics, population, measurement tools, monitoring process, and measured outcomes. Study metrics refer to the name of authors, article title, year of publication, journal's name, country of study, study design, number of centers taking part in the study, funding, and conflicts of interest. Population-related information includes sample size, age, gender, weight, height, and BMI for both control and patient populations. Information about occupational status, education, and lifestyle (smoking and alcohol use) will be extracted. Data about disease qualification, disease duration, and severity will also be extracted. Data related to measurement tools will be included, that is, the device and manufacturer name, the type of embedded sensors, the wearing location, and the properties of the devices (restriction about their usage and particularity of the device). Experimental procedure data will be reported through the length of monitoring, the days included in the monitoring, and the wear time instructions. Reported outcomes will be step count, gait speed, or parameters relative to walking activity. Conclusions and clinical or research implications will also be extracted. If possible, a meta-analysis will be conducted highlighting the research findings. In case of missing or erroneous data, the study authors will be contacted for further information. Discussion and consensus meeting will be used to resolve discrepancy.

\section{Data Synthesis}

This review is specifically designed to present an overview of the literature existing on the assessment of daily walking activity in people with PD. This review establishes a synthesis of the existing methods and devices already in use to assess walking activity in PD patients. We will detail and summarize the common usage concerning the wearable devices used in this population. This review will present the major trend in terms of wearing location and tools used as well as the methodology used to capture daily walking activity. These data will be presented categorized according to the population and study characteristics, the wearable devices, the monitoring methods, and the reported outcomes.

\section{Results}

A flowchart will be constructed depicting each stage of the inclusion process and the corresponding number of articles selected with the exception of the nonmandatory risk of bias and strength of evidence assessments [37].

\section{Discussion}

Due to the particularity of the walking activities, we expect only minor heterogeneity concerning the reported outcomes. However, we expect a wide heterogeneity regarding the methodology deployed to track walking activity. Based on the initial screening process, we identified 3 main types of studies that would be included in this review.

First, the studies aiming to assess the effectiveness of the use of a wearable device during intervention focusing on physical activity, balance, or gait. These studies frequently provide a daily step count, reported at baseline and at the end of the intervention, as a measure of gait stability or physical activity level.

Second, studies aiming to assess the performance of different wearable devices in patients with PD. Some of them focusing on the on-body location effect while others assessing the feasibility of using wearables as a tool to track activity in PD patients. In this type of study, step count is frequently reported as a measure of accuracy, and it is often compared to a criterion measure.

Third, studies aiming to approach the walking specificity of patients with PD with wearable devices. In such studies, wearables are used as a noninvasive tool to assess disease severity or implication, and step count also is reported as an indirect measure of disease severity.

Due to these differences in terms of the use of step count in addition to the wide variability in study design and devices, we decided to conduct a narrative synthesis; however, depending on the number of studies with the same design and with the same outcomes, we will not reject the possibility to perform a meta-analysis of the daily walking activity in patients with PD. 


\section{Conclusion}

This review will present researchers and clinicians with an overview of all studies assessing the walking activity in patients with PD. Recent technological advances allow researchers and clinicians to capture long-term behavior change [18, 27] and individual activity patterns using wearable devices [39-41]. This review aims to clarify and synthesize the evidence about wearable device use in patients with PD to assess walking activity with a focus on device selection.

\section{Acknowledgment}

We acknowledge financial support by DFG within the funding program Open Access Publizieren.

\section{Statement of Ethics}

As this review is limited to publicly available materials, it does not require ethical approval. Results will be shared with the scientific community and general public.

\section{Conflict of Interest Statement}

The authors have no conflicts of interest to declare.

\section{Funding Sources}

This work was supported by the French National Research Agency in the framework of the "Investissements d'avenir" program (ANR-10-AIRT-05 and ANR-15-IDEX-02). This work further forms part of a broader translational and interdisciplinary research project, GaitAlps (N Vuillerme).

\section{Author Contributions}

T.C., C.H., and N.V. devised study scope and research questions and contributed to study design. T.C., C.H., and N.V. wrote and edited the manuscript. T.C. and C.H. will review references and extract data. All authors approved the final manuscript.

\section{References}

1 Gelb DJ, Oliver E, Gilman S. Diagnostic criteria for Parkinson disease. Arch Neurol. 1999 Jan 1;56(1):33.

2 Poewe W, Seppi K, Tanner CM, Halliday GM, Brundin P, Volkmann J, et al. Parkinson disease. Nat Rev Dis Primers. 2017 Dec;3(1): 17013.

3 Chaudhuri KR, Healy DG, Schapira AHV; National Institute for Clinical Excellence. Non-motor symptoms of Parkinson's disease: diagnosis and management. Lancet Neurol. 2006 Mar;5(3):235-45.

4 Lang AE, Lozano AM. Parkinson's disease. Second of two parts. N Engl J Med. 1998 Oct 15;339(16):1130-43.

5 Breen DP, Lang AE. Tracking the course of prodromal Parkinson's disease. Brain. 2017 Feb;140(2):259-62.

6 Darweesh SK, Verlinden VJ, Stricker BH, Hofman A, Koudstaal PJ, Ikram MA. Trajectories of prediagnostic functioning in Parkinson's disease. Brain. 2017 Feb;140(2):429-41.

7 Wolters EC. Variability in the clinical expression of Parkinson's disease. J Neurol Sci. 2008 Mar;266(1-2):197-203.

8 Caslake R, Taylor K, Scott N, Gordon J, Harris C, Wilde K, et al. Age-, gender-, and socioeconomic status-specific incidence of Parkinson's disease and parkinsonism in North East Scotland: the PINE study. Parkinsonism Relat Disord. 2013 May;19(5):515-21.
9 de Lau LML, Verbaan D, Marinus J, van Hilten JJ. Survival in Parkinson's disease. Relation with motor and non-motor features. Parkinsonism Relat Disord. 2014 Jun;20(6): 613-6.

10 Dickson JM, Grünewald RA. Somatic symptom progression in idiopathic Parkinson's disease. Parkinsonism Relat Disord. 2004 Dec;10(8):487-92.

11 Szewczyk-Krolikowski K, Tomlinson P, Nithi K, Wade-Martins R, Talbot K, Ben-Shlomo Y, et al. The influence of age and gender on motor and non-motor features of early Parkinson's disease: initial findings from the Oxford Parkinson Disease Center (OPDC) discovery cohort. Parkinsonism Relat Disord. 2014 Jan; 20(1):99-105.

12 Savitt JM, Dawson VL, Dawson TM. Diagnosis and treatment of Parkinson disease: molecules to medicine. J Clin Invest. 2006 Jul 3; 116(7):1744-54.

13 Glendinning D. A rationale for strength training in patients with Parkinson's disease. Neurology Report. 1997;21(4):132-5.

14 Li F, Harmer P, Fitzgerald K, Eckstrom E, Stock R, Galver J, et al. Tai chi and postural stability in patients with Parkinson's disease. N Engl J Med. 2012 Feb 9;366(6):511-9.

15 Chung CLH, Thilarajah S, Tan D. Effectiveness of resistance training on muscle strength and physical function in people with Parkinson's disease: a systematic review and metaanalysis. Clin Rehabil. 2016 Jan;30(1):11-23.
16 Bridgewater KJ, Sharpe MH. Trunk muscle training and early Parkinson's disease. Phys Ther. 1997 Jan;13(2):139-53.

17 Nimwegen M, Speelman AD, Hofman-van Rossum EJM, Overeem S, Deeg DJH, Borm GF, et al. Physical inactivity in Parkinson's disease. J Neurol. 2011 Dec;258(12):2214-21.

18 Speelman AD, van de Warrenburg BP, van Nimwegen M, Petzinger GM, Munneke M, Bloem BR. How might physical activity benefit patients with Parkinson disease? Nat Rev Neurol. 2011 Sep;7(9):528-34.

19 Allen NE, Schwarzel AK, Canning CG. Recurrent falls in Parkinson's disease: a systematic review. Parkinsons Dis. 2013;2013:1-16.

20 Bloem BR, Grimbergen YA, Cramer M, Willemsen $\mathrm{M}$, Zwinderman AH. Prospective assessment of falls in Parkinson's disease. J Neurol. 2001 Nov 1;248(11):950-8.

21 Kerr GK, Worringham CJ, Cole MH, Lacherez PF, Wood JM, Silburn PA. Predictors of future falls in Parkinson disease. Neurology. 2010 Jul 13;75(2):116-24.

22 Chen H, Zhang SM, Schwarzschild MA, Hernán MA, Ascherio A. Physical activity and the risk of Parkinson disease. Neurology. 2005 Feb 22;64(4):664-9.

23 Wu P-L, Lee M, Huang T-T. Effectiveness of physical activity on patients with depression and Parkinson's disease: a systematic review. Lebedev MA, editor. PLoS One. 2017 Jul 27; 12(7):e0181515.
Wearable Technology to Measure

Walking Activity 
24 Cusso ME, Donald KJ, Khoo TK. The impact of physical activity on non-motor symptoms in Parkinson's disease: a systematic review. Front Med. 2016 Aug 17;3:35. Available from: http: //journal.frontiersin.org/Article/10.3389/ fmed.2016.00035/abstract.

25 Galperin I, Hillel I, Del Din S, Bekkers EMJ, Nieuwboer A, Abbruzzese G, et al. Associations between daily-living physical activity and laboratory-based assessments of motor severity in patients with falls and Parkinson's disease. Parkinsonism Relat Disord. 2019 May;62:85-90.

26 Goodwin VA, Richards SH, Taylor RS, Taylor AH, Campbell JL. The effectiveness of exercise interventions for people with Parkinson's disease: a systematic review and meta-analysis: PD and exercise. Mov Disord. $2008 \mathrm{Apr}$ 15;23(5):631-40.

27 Speelman AD, van Nimwegen M, Bloem BR, Munneke M. Evaluation of implementation of the ParkFit program: a multifaceted intervention aimed to promote physical activity in patients with Parkinson's disease. Physiotherapy. 2014 Jun;100(2):134-41.

28 Rovini E, Maremmani C, Cavallo F. How wearable sensors can support Parkinson's disease diagnosis and treatment: a systematic review. Front Neurosci. 2017 Oct 6;11:555.
29 Kenyon A, McEvoy M, Sprod J, Maher C. Validity of pedometers in people with physical disabilities: a systematic review. Arch Phys Med Rehabil. 2013 Jun;94(6):1161-70.

30 Cavanaugh JT, Ellis TD, Earhart GM, Ford MP, Foreman KB, Dibble LE. Capturing ambulatory activity decline in Parkinson's disease. J Neurol Phys Ther. 2012 Jun;36(2):51-7.

31 Skidmore FM, Mackman CA, Pav B, Shulman LM, Garvan C, Macko RF, et al. Daily ambulatory activity levels in idiopathic Parkinson disease. J Rehabil Res Dev. 2008;45(9):1343.

32 Prajapati SK, Gage WH, Brooks D, Black SE, McIlroy WE. A novel approach to ambulatory monitoring: investigation into the quantity and control of everyday walking in patients with subacute stroke. Neurorehabil Neural Repair. 2011 Jan;25(1):6-14.

33 Warmerdam E, Hausdorff JM, Atrsaei A, Zhou Y, Mirelman A, Aminian K, et al. Longterm unsupervised mobility assessment in movement disorders. Lancet Neurol. 2020; 19(5):462-70.

34 Creaby MW, Cole MH. Gait characteristics and falls in Parkinson's disease: a systematic review and meta-analysis. Parkinsonism Relat Disord. 2018;57:1-8.

35 Bryant MS, Rintala DH, Hou JG, Charness AL, Fernandez AL, Collins RL, et al. Gait variability in Parkinson's disease: influence of walking speed and dopaminergic treatment. Neurol Res. 2011 Nov;33(9):959-64.
36 Erb MK, Karlin DR, Ho BK, Thomas KC, Parisi F, Vergara-Diaz GP, et al. mHealth and wearable technology should replace motor diaries to track motor fluctuations in Parkinson's disease. NPJ Digit Med. 2020;3:6.

37 Moher D, Liberati A, Tetzlaff J, Altman DG; The PRISMA Group. Preferred reporting items for systematic reviews and meta-analyses: the PRISMA statement. Open Med. 2009 Jul 21;3(7):e123.

38 Tricco AC, Lillie E, Zarin W, O'Brien KK, Colquhoun $\mathrm{H}$, Levac D, et al. PRISMA extension for scoping reviews (PRISMA-ScR): checklist and explanation. Ann Intern Med. 2018 Oct 2;169(7):467-73.

39 Saint-Maurice PF, Troiano RP, Bassett DR, Graubard BI, Carlson SA, Shiroma EJ, et al. Association of daily step count and step intensity with mortality among US adults. JAMA. 2020 Mar 24;323(12):1151.

40 Bassett DR, Toth LP, LaMunion SR, Crouter SE. Step counting: a review of measurement considerations and health-related applications. Sports Med. 2017 Jul;47(7):1303-15.

41 Strain T, Wijndaele K, Dempsey PC, Sharp SJ, Pearce M, Jeon J, et al. Wearable-device-measured physical activity and future health risk. Nat Med. 2020 Sep;26(9):1385-91. 